\title{
Legumain promotes tubular ferroptosis by facilitating chaperone-mediated autophagy of GPX4 in AKI
}

\author{
Chuan'ai Chen', Dekun Wang ${ }^{1}$, Yangyang Yu', Tianyuan Zhao', Ningning Min ${ }^{1}$, Yan Wu', Lichun Kang ${ }^{1}$, Yong Zhao', \\ Lingfang Du', Mianzhi Zhang ${ }^{2}$, Junbo Gong ${ }^{3}$, Zhujun Zhang ${ }^{1}$, Yuying Zhang ${ }^{1}$, Xue Mi', Shijing Yue $\mathbb{B}^{1}$ and \\ Xiaoyue $\operatorname{Tan}^{1}$
}

\begin{abstract}
Legumain is required for maintenance of normal kidney homeostasis. However, its role in acute kidney injury (AKI) is still unclear. Here, we induced AKI by bilateral ischemia-reperfusion injury (IRI) of renal arteries or folic acid in lgmn ${ }^{\text {WT }}$ and $\mathrm{lgm} n^{\mathrm{KO}}$ mice. We assessed serum creatinine, blood urea nitrogen, histological indexes of tubular injury, and expression of KIM-1 and NGAL. Inflammatory infiltration was evaluated by immunohistological staining of CD3 and F4/ 80, and expression of TNF-a, CCL-2, IL-33, and IL-1a. Ferroptosis was evaluated by Acs $\mid 4$, Cox-2, reactive oxygen species (ROS) indexes $\mathrm{H}_{2} \mathrm{DCFDA}$ and DHE, MDA and glutathione peroxidase 4 (GPX4). We induced ferroptosis by hypoxia or erastin in primary mouse renal tubular epithelial cells (mRTECs). Cellular survival, Acs/4, Cox-2, LDH release, ROS, and MDA levels were measured. We analyzed the degradation of GPX4 through inhibition of proteasomes or autophagy. Lysosomal GPX4 was assessed to determine GPX4 degradation pathway. Immunoprecipitation (IP) was used to determine the interactions between legumain, GPX4, HSC70, and HSP90. For tentative treatment, RR-11a was administrated intraperitoneally to a mouse model of IRI-induced AKI. Our results showed that legumain deficiency attenuated acute tubular injury, inflammation, and ferroptosis in either IRI or folic acid-induced AKI model. Ferroptosis induced by hypoxia or erastin was dampened in $\operatorname{lgm} n^{\mathrm{KO}}$ mRTECs compared with $\lg m n^{\mathrm{WT}}$ control. Deficiency of legumain prevented chaperone-mediated autophagy of GPX4. Results of IP suggested interactions between legumain, HSC70, HSP90, and GPX4. Administration of RR-11a ameliorated ferroptosis and renal injury in the AKI model. Together, our data indicate that legumain promotes chaperone-mediated autophagy of GPX4 therefore facilitates tubular ferroptosis in AKI.
\end{abstract}

\section{Introduction}

Acute kidney injury (AKI) is a severe clinical syndrome manifested by rapid and possibly transient loss of renal functions ${ }^{1}$. Because of an aging population, increasing incidences of cardiovascular diseases, diabetes mellitus, and chronic kidney diseases, as well as expanding

\footnotetext{
Correspondence: Xiaoyue Tan (xiaoyuetan@nankai.edu.cn)

${ }^{1}$ School of Medicine, State Key Laboratory of Medicinal Chemical Biology,

Nankai University, Tianjin 300071, China

${ }^{2}$ Dongfang Hospital of Beijing University of Chinese Medicine, Beijing 100078, China

Full list of author information is available at the end of the article

Edited by A. Linkermann
}

characterization of risk factors including sepsis, exposure to contrast media, and other nephrotoxins, the incidence of AKI is continuously increasing 2,3 . There is still no satisfactory treatment to attenuate AKI or accelerate recovery, resulting in mortality of AKI as high as $50 \%$. Elucidating the regulatory mechanisms underlying the loss and repair of tubular cells during AKI is required to develop effective therapeutic strategies and improve prognosis.

Tubular cell death is the major early event during the process of AKI, which is accompanied by inflammation due to chemokines and damage-associated molecular

\section{(c) The Author(s) 2021}

(c) (i) Open Access This article is licensed under a Creative Commons Attribution 4.0 International License, which permits use, sharing, adaptation, distribution and reproduction cc) in any medium or format, as long as you give appropriate credit to the original author(s) and the source, provide a link to the Creative Commons license, and indicate if changes were made. The images or other third party material in this article are included in the article's Creative Commons license, unless indicated otherwise in a credit line to the material. If material is not included in the article's Creative Commons license and your intended use is not permitted by statutory regulation or exceeds the permitted use, you will need to obtain permission directly from the copyright holder. To view a copy of this license, visit http://creativecommons.org/licenses/by/4.0/. 
patterns from the dead and dying cells ${ }^{5}$. Distinct forms of cell death have been indicated to participate in the loss of tubular cells in $\mathrm{AKI}^{2,6}$. Ferroptosis, a newly recognized form of programmed cell death characterized by iron-dependent lipid hydroperoxide accumulation to lethal levels, has been reported to be of pathophysiological relevance in $\mathrm{AKI}^{7-9}$. In mouse models of AKI induced by either ischemia-reperfusion injury (IRI) or oxalate crystal, ferroptosis is directly involved in the synchronized necrosis of renal tubules ${ }^{10}$. Ferroptosis and its subsequent immunogenicity play the primary role in the kidney damage of folic acid-induced $\mathrm{AKI}^{11}$. Among the known regulatory factors involved in ferroptosis, GPX4 is the critical enzyme that reduces phospholipid hydroperoxides, acting as a gatekeeper for ferroptosis through selectively detoxifying lipid hydroperoxides $^{12}$. Genetically inactive GPX4 leads to tubular cell death in a pathologically relevant form of ferroptosis and the occurrence of lipid-oxidation-induced $\mathrm{AKI}^{13}$. Modulating GPX4 abundance or activity results in the initiation and suppression of ferroptosis or sensitizing cells to ferroptosis ${ }^{14,15}$. A recent study revealed that activation of chaperone-mediated autophagy (CMA) was involved in the degradation of GPX4, and stabilizing GPX4 by inhibition of CMA reduced ferroptosis ${ }^{16}$. In this study, we found that asparaginyl endopeptidase legumain was involved in the degradation of GPX4 through CMA, affecting the process of ferroptosis during the progression of AKI.

Legumain is a conserved asparaginyl endopeptidase that is highly expressed in proximal tubular cells in the physiological state ${ }^{17}$. The birth and outward appearance of legumain knockout mice is normal. Renal histological abnormalities were first evident around 2 months of life, including hyperplasia of proximal tubular cells and abnormal mitotic figures. Interstitial fibrosis appeared by 3 months and glomerular cysts became evidence around 10 months. Glomerular filtration rate falls with increased urine albumin occurred in 6-month-old knockout mice, implying legumain is indispensable for the maintenance of normal kidney physiology and homeostasis ${ }^{18}$. Our previous data have demonstrated that M2 macrophagederived legumain promotes degradation of the extracellular matrix, resulting in an anti-fibrotic effect in a mouse model of obstructive nephropathy. However, the context-dependent role of legumain during AKI is still unclear. In this study, through genetic engineering of murine models, we found that legumain deficiency ameliorated ferroptosis of tubular cells in AKI induced by either IRI or nephrotoxic folic acid. Furthermore, the beneficial role of legumain deficiency was mediated by the maintenance of cytosolic GPX4. We also found that legumain interacted with GPX4 and facilitated its autophagic degradation.
Taken together, our findings revealed that legumain is involved in the pathogenesis of AKI via regulating the degradation of major ferroptosis-protective factor GPX4, suggesting legumain as a therapeutic target for the treatment of AKI.

\section{Results \\ Deletion of legumain appears renal protective effects in mice with renal IRI}

Renal proximal tubular cells highly express legumain in the physiological state. To evaluate the effect of legumain in IRI-induced AKI, lgmn knockout mice (hereafter referred to as $\left.\operatorname{lgm} n^{\mathrm{KO}}\right)$ and littermate control $\left(\operatorname{lgm} n^{\mathrm{WT}}\right)$ mice were subjected to 40 minutes of bilateral renal ischemia or sham surgery. BUN, serum creatinine, renal tubular injury, and transcript levels of KIM-1 and NGAL were measured before and at 1,2 , and 7 days after reperfusion. A slight decrease of legumain was observed in $\operatorname{lgmn} n^{\mathrm{WT}}$ kidneys at day 1 after reperfusion, with an increase at day 2 and returned to the baseline level at day 7 (Supplementary Figure 1A). Compared with lgmn WT control, IRI-induced increases in BUN and serum creatinine at 1 and 2 days after reperfusion were attenuated in lgmn ${ }^{\mathrm{KO}}$ mice (Fig. 1A, B). Similarly, induced mRNA expression of molecular markers of tubular injury, KIM-1 and NGAL was also suppressed in $l g m n^{\mathrm{KO}}$ mice (Fig. $1 \mathrm{C}$, D). Based on the detachment and dilation of renal tubules and brush border damage shown by PAS staining, acute tubular necrosis (ATN) score was calculated. We found that indexes of tubular injury and ATN score at 1 and 2 days after reperfusion were decreased in the $\operatorname{lgm} n^{\mathrm{KO}}$ group compared with lgmn ${ }^{\mathrm{WT}}$ mice (Fig. 1E-G).

Inflammatory cell infiltration plays a key role in the pathogenesis of IRI-induced tubular lesions. Therefore, we compared the extent of inflammation in the IRI model of $\operatorname{lgm} n^{\mathrm{WT}}$ and $\operatorname{lgm} n^{\mathrm{KO}}$ mice. CD3-positive T cells and F4/ 80-positive macrophages were measured by immunohistological staining. Expression of proinflammatory cytokines TNF- $\alpha, C C L 2, I L-33$, and $I L-1 \alpha$ was assessed. Our data showed that IRI-induced increase of inflammatory responses, including infiltration of inflammatory cells (Fig. 2A, B), and increase of inflammatory cytokines (Fig. $2 \mathrm{C}-\mathrm{G})$ were suppressed in $\operatorname{lgm} n^{\mathrm{KO}}$ kidneys compared with control. Therefore, our data provide evidences that legumain promotes acute tubular lesions and inflammatory reactions induced by IRI.

\section{Legumain-deficient IRI mice exhibit reduced renal ferroptosis}

Multiple insults result in tubular cell death in the context of IRI, including apoptosis, prominent programmed, and unprogrammed necrosis. It is recognized that ferroptosis, a unique form of programmed cell death characterized by iron-dependent and excess lipid 


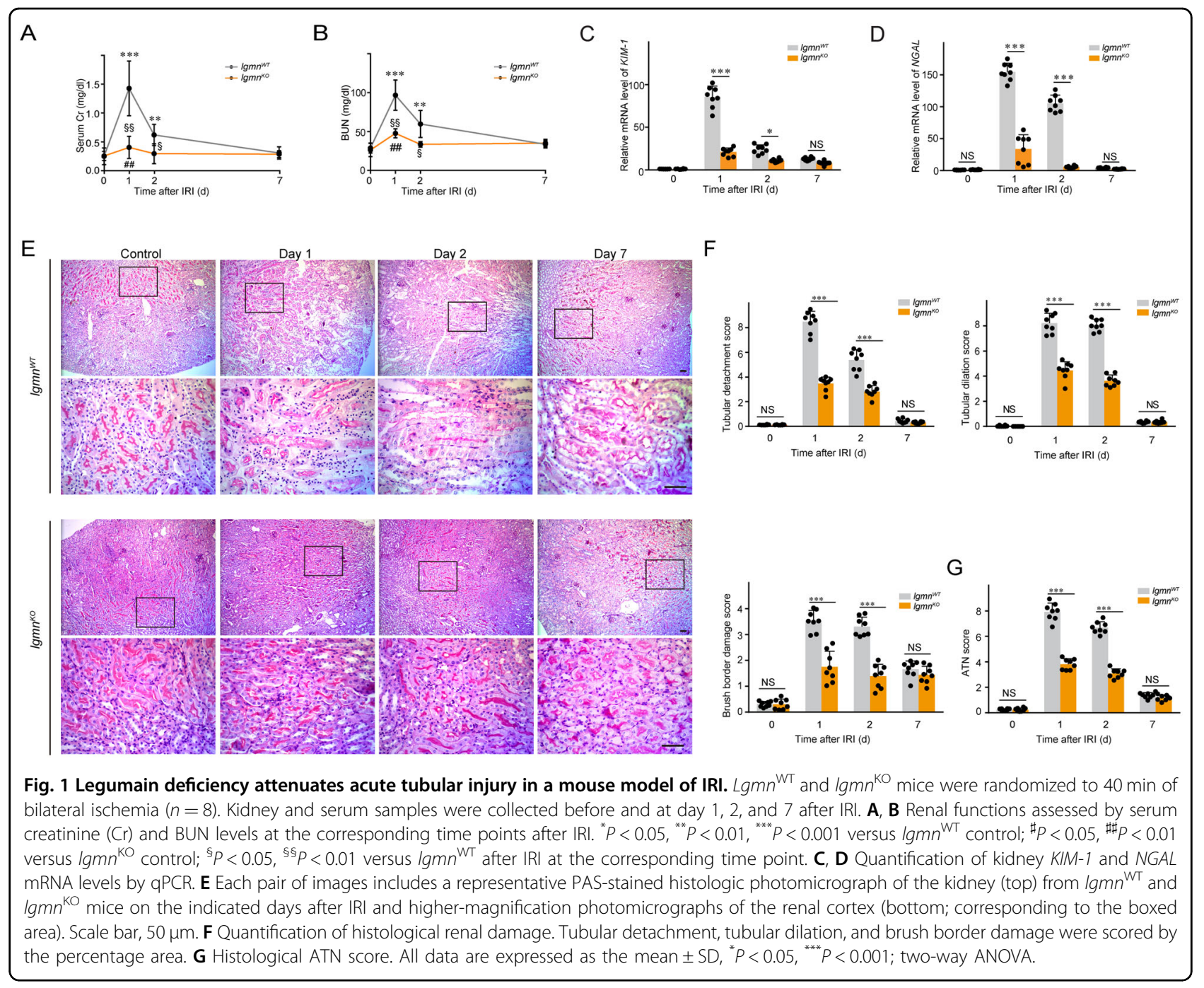

peroxidation, contributes to renal tubular cell loss during AKI. To identify the role of ferroptosis in the renoprotective role of legumain knockout in the IRI model, we assessed indexes of ferroptosis, including products of lipid peroxidation, ROS, and degradation of phospholipid peroxidase GPX4. We found that Acsl4 and Cox-2, two enzymes responsible for the biosynthesis of ferroptosissensitive phospholipids, were inhibited in IRI $\operatorname{lgm} n^{\mathrm{KO}}$ mice compared with control (Fig. 3A, B). Cytosolic ROS sensor carboxy- $\mathrm{H}_{2}$ DCFDA and DHE were used to characterize intracellular ROS, and MDA content was measured to evaluate the end products of lipid peroxidation. Our results showed that ROS (Fig. 3C, D) and lipid peroxidation (Fig. 3E) were attenuated in $\operatorname{lgm} n^{\mathrm{KO}}$ mice compared with control. Degradation of GPX4, a wellknown inhibitory enzyme of lipid peroxidation is critical for driving ferroptosis. To determine whether legumain deficiency affected the transcription level of GPX4, we measured GPX4 mRNA in kidney samples and found that
IRI did not influence mRNA level of GPX4 in $\operatorname{lgm} n^{\mathrm{KO}}$ or $\operatorname{lgmn}{ }^{\mathrm{WT}}$ mice (Fig. 3F). However, IRI induced a reduction of GPX4 protein in lgmn ${ }^{\mathrm{WT}}$ group, which was blocked in $\operatorname{lgmn} n^{\mathrm{KO}}$ mice (Fig. 3G). As for the markers of apoptosis and necroptosis, we found that there was no significant difference on cleaved caspase 3 at 1,2, or 7-day of IRI and $\mathrm{p}-\mathrm{MLKL}$ at 1 or 7 -day of IRI between $\operatorname{lgm} n^{\mathrm{WT}}$ and $\lg m n^{\mathrm{KO}}$ mice, although less p-MLKL could be detected on day 2 of IRI in the $\operatorname{lgm}{ }^{\mathrm{KO}}$ mice. (Supplementary Figure 2A, B). Collectively, these results indicate that legumain deficiency inhibits ferroptosis induced by IRI.

\section{Downregulation of legumain inhibits ferroptosis in renal tubular cells}

To further identify whether legumain deficiency protects renal tubular cells against ferroptosis, we cultured primary mRTECs isolated from $\operatorname{lgm} n^{\mathrm{WT}}$ and $\operatorname{lgm} n^{\mathrm{KO}}$ mice. $\operatorname{lgm} n^{\mathrm{WT}}$ and $\operatorname{lgm} n^{\mathrm{KO}}$ mRTECs were treated with ferroptosis inducer erastin or simulated by hypoxia. Our 


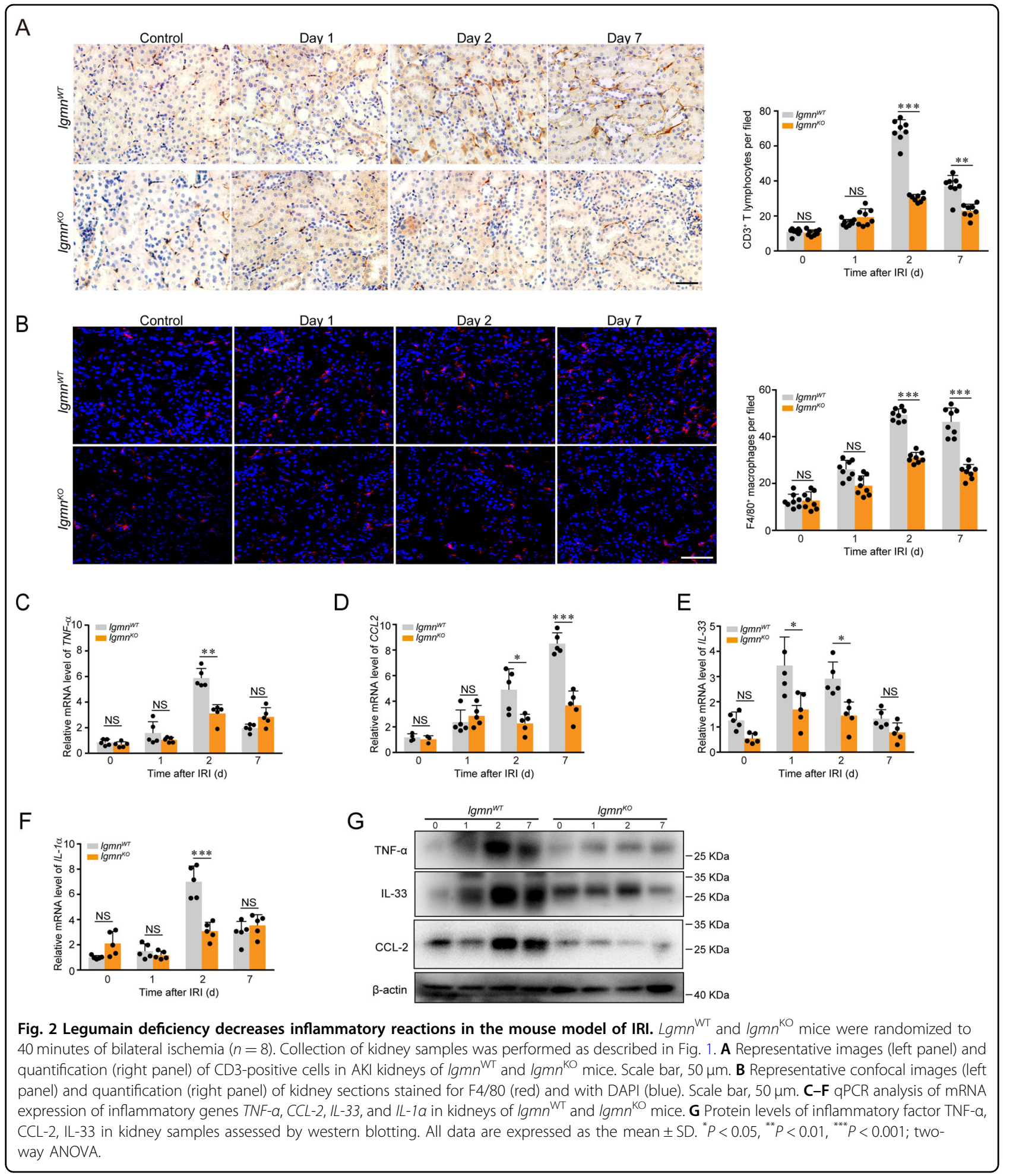

results showed that both erastin and hypoxic stimuli induced legumain in mRTECs (Supplementary Figure 1B, C). Legumain knockout rescued erastin-induced decrease of cell survival and expression of Acsl 4 and Cox-2 mRNAs (Fig. 4A, B). Increased MDA induced by erastin was also rescued after legumain knockout (Fig. 4C). Similarly, hypoxia reduced cell survival and increased LDH release, but these effects were blocked by knockout of legumain (Fig. 4D, E). Inhibitors of ferroptosis Fer-1 and iron chelator DFO rescued cell death induced by hypoxia of 12 


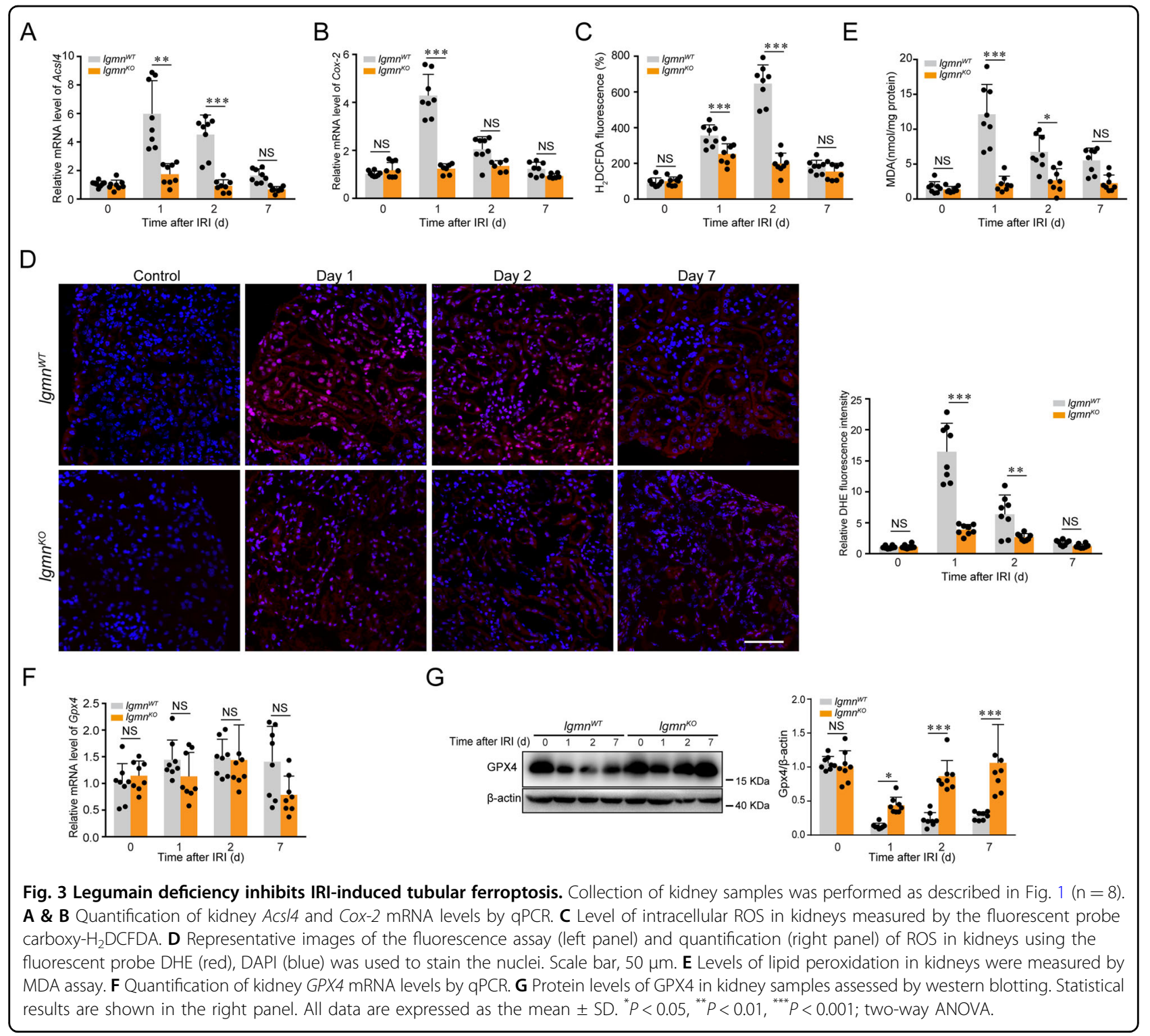

and $24 \mathrm{~h}$, while inhibitor of necroptosis Nec-1 only partially protect cell death from hypoxic injury of $24 \mathrm{~h}$ (Fig. $4 \mathrm{~F}$ ), indicating ferroptosis contributing to the hypoxiainduced cell death. We then detected ferroptosis indexes in $\operatorname{lgmn}^{\mathrm{WT}}$ and $\operatorname{lgmn}^{\mathrm{KO}}$ mRTECs stimulated by hypoxia. We found that hypoxia not only increased expression of Acsl4 and Cox-2 mRNAs, but also level of ROS and MDA. Compared with wildtype control, Acsl4 and Cox-2 mRNA expression (Fig. 4G) and level of ROS and MDA were reduced in $\operatorname{lgmn}^{\mathrm{KO}}$ mRTECs (Fig. 4H, I). Similarly, legumain knockout mitigated ferroptosis induce by erastin or RSL3 in 786-O and OSRC-2, two renal tubular carcinoma cell lines (Supplementary Figure 4). Taken together, our data indicate that downregulation of legumain inhibits erastin- and hypoxia-induced ferroptosis in renal tubular cells.
Legumain participates in lysosomal degradation of GPX4 during ferroptosis

Given that legumain plays a role in tubular ferroptosis, we further explored the underlying molecular mechanism. Both erastin and hypoxia inhibited GPX4 protein in wildtype primary tubular cells, and this effect was rescued in legumain knockout cells (Fig. 5A). No significant difference of GPX4 mRNA was found between legumain knockout and wildtype cells (Fig. 5B), suggesting that the regulatory effect of legumain on GPX4 occurs at protein level. Various protein degradation inhibitors, including proteasome and lysosome inhibitors, were used to evaluate the role of distinct degradation pathways in the legumain-related change of GPX4 level. Treatment with lysosome inhibitors chloroquine or bafilomycin A1, but not proteasome inhibitor MG-132, rescued the decrease 


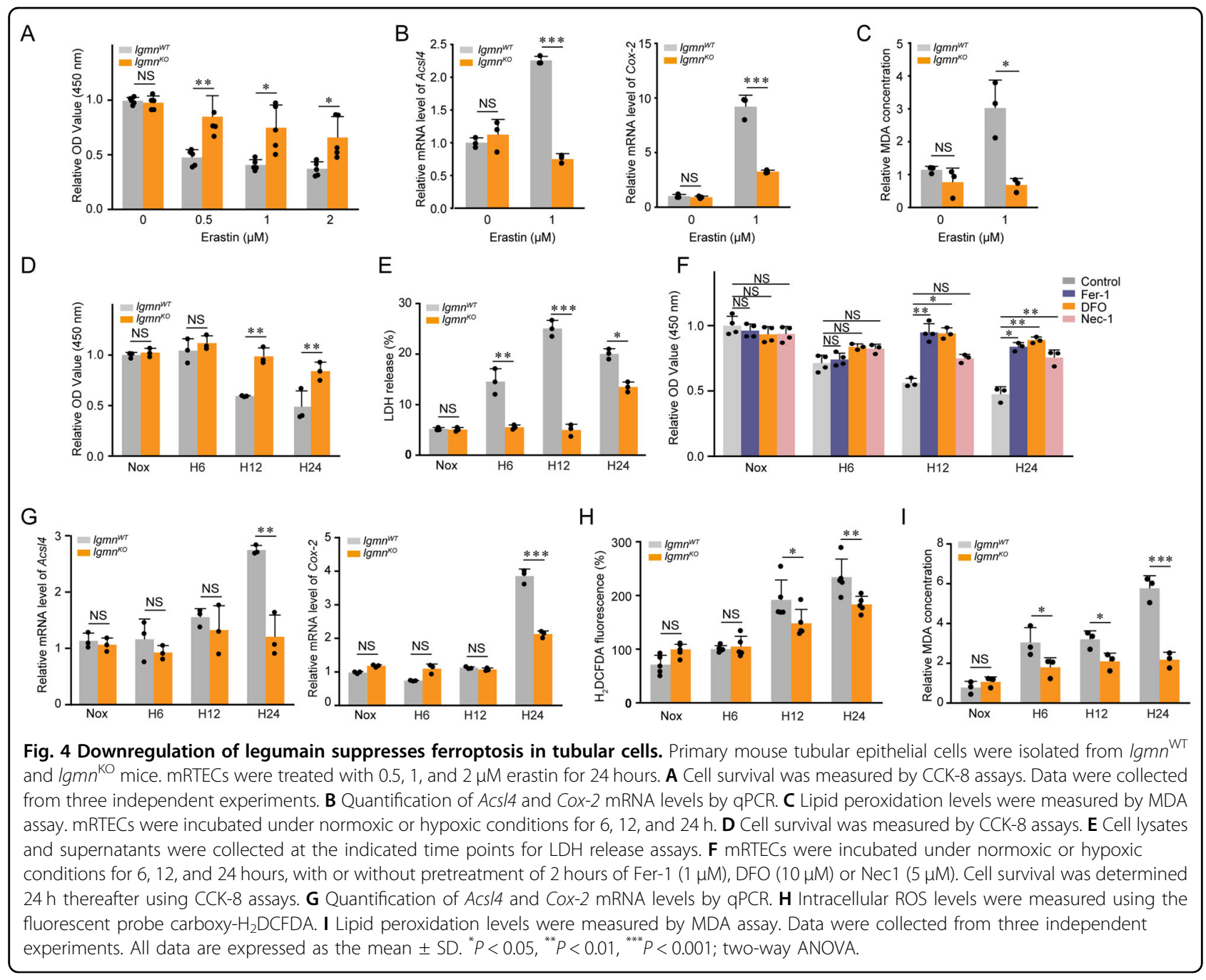

of erastin-induced GPX4 (Fig. 5C). Similar results were found in erastin or RSL3 induced ferroptosis in 786-O and OSRC-2 cells (Supplementary Figure 5). These results suggest that the protective effect of legumain deficiency on GPX4 dependents on inhibition of its lysosomal degradation.

We further compared GPX4 in lysosomes and other cellular compartments during erastin- and hypoxiainduced ferroptosis in $\operatorname{lgm} n^{\mathrm{KO}}$ and $\operatorname{lgm} n^{\mathrm{WT}}$ mRTECs. Our results showed that knockout of legumain abolished increase of lysosomal GPX4 induced by erastin or hypoxia (Fig. 5D). Moreover, overexpression of legumain blocked the change of GPX4 induced by legumain knockout (Fig. $5 \mathrm{E}, \mathrm{F})$. A previous study has indicated that activation of ferroptosis leads to increases of HSC70-mediated lysosomal delivery and degradation of GPX4. Therefore, we hypothesized that cytosolic legumain participated in the chaperone-mediated lysosomal transport of GPX4. Immunoprecipitation assays suggested interactions between legumain, HSP90, HSC70, lamp-2a and GPX4
(Fig. 5G). These interactions were increased by hypoxia compared with control (Fig. 5H). Therefore, our data provide evidences that cytosolic legumain interacts with HSC70, HSP90, lamp-2a and GPX4, mediating lysosomal transport of GPX4 and subsequently ferroptosis.

\section{Legumain deficiency attenuates tubular ferroptosis in folic acid-induced AKI}

We next investigated the effect of legumain deficiency on nephrotoxic folic acid model in which ferroptosis has been proven to be critical for the pathogenesis of AKI. We found that legumain was increased in 24 and $48 \mathrm{~h}$ of folic acid-induced AKI model (Supplementary Figure 6A, B). In vitro, treatment of folic acid increased level of legumain in cultured mRTECs (Supplementary Figure 6C). Compared with wildtype control, lower levels of serum creatinine and BUN were found in $\operatorname{lgmn}^{\mathrm{KO}}$ mice (Fig. 6A). Consistently, folic acid-induced increases of KIM-1 and NGAL expression were also suppressed in $\operatorname{lgm} n^{\mathrm{KO}}$ mice (Fig. 6B). Dilation of renal tubules, brush border damage, and the 


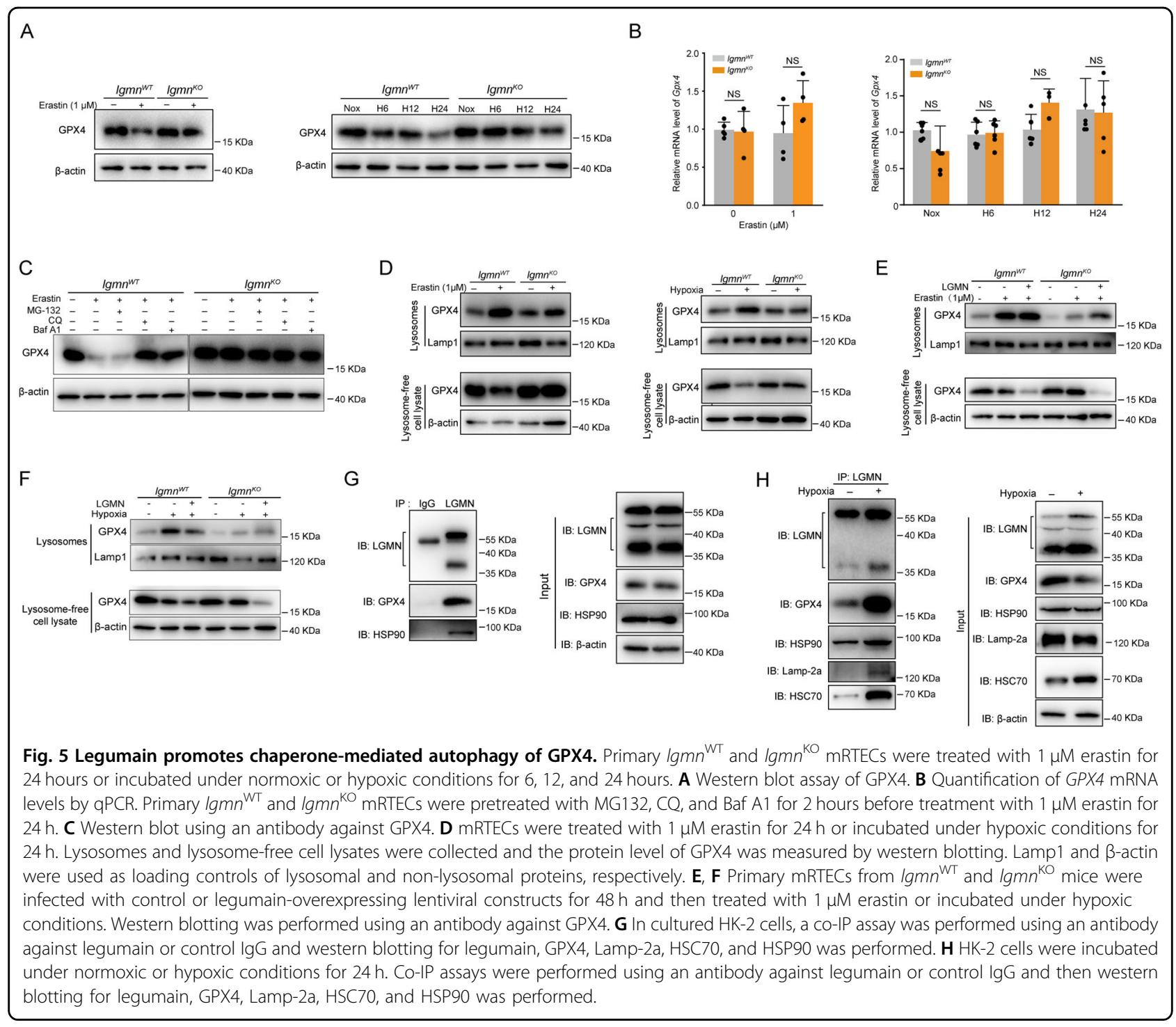

protein cast percentage assessed by PAS staining showed similar results, indicating that knockout of legumain attenuated folic acid-induced tubular injury (Fig. 6C, D). Indexes of ferroptosis, including expression of Acsl4 and Cox-2 mRNAs and level of MDA, were significantly increased after 24 and 48 hours of treatment of folic acid. Compared with control, both Acsl4 and Cox-2 mRNAs and level of MDA were reduced in knockout mice (Fig. 6E, F). The decreased GPX4 level in kidney tissue after 24 and 48-hour of treatment with folic acid was reserved in knockout mice compared with control (Fig. 6G). In mRTECs isolated from the folic acid-induced AKI model, similar changes in GPX4 expression were found. Specifically, the change in the GPX4 level of $\operatorname{lgm} n^{\mathrm{KO}}$ mRTECs after 24 and 48-hour of folic acid treatment was reserved compared with the control (Fig. 6H). Thus, our data demonstrate that knockout of legumain also protects against tubular lesions and ferroptosis caused by nephrotoxic folic acid.

\section{RR-11a ameliorates ferroptosis and acute tubular injury in the IRI model}

To investigate the potential application of legumain inhibitor in AKI, we firstly confirmed the inhibitory effect of RR-11a on ferroptosis of mRTECs induced by hypoxia in vitro. (Fig. 7A-C). Then we determined whether administration of RR-11a ameliorated acute tubular injury in the IRI model. At 1 day after IRI, levels of serum creatinine and BUN were significantly lower in the RR11a group compared with the vehicle control (Fig. 7D, E). Indexes of tubular injury, including mRNA expression of KIM-1 and NGAL and the histological ATN score were improved in the RR-11a group (Fig. 7F-I). To examine changes in ferroptosis, we compared mRNA expression of 


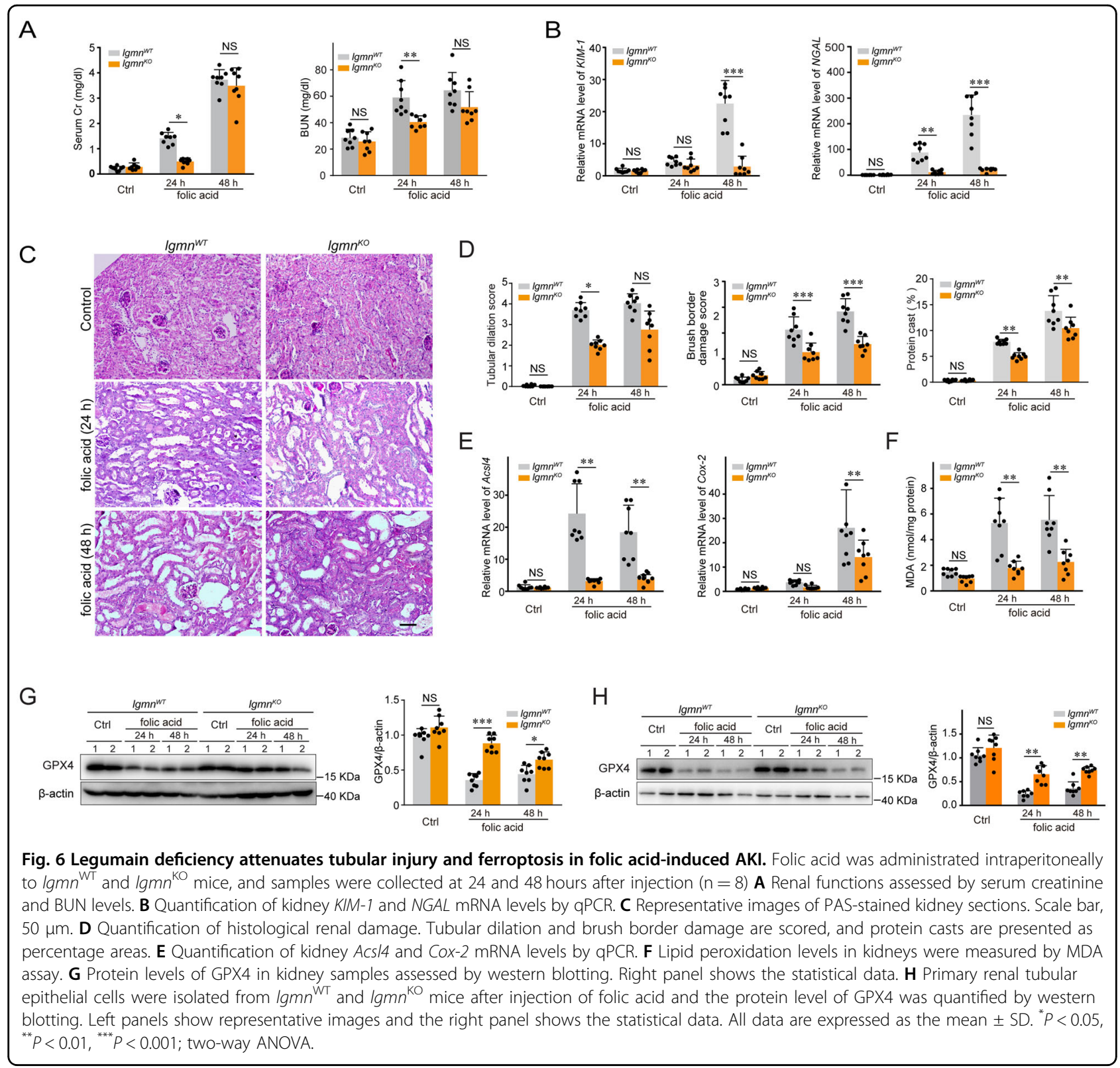

Acsl4, Cox-2, and GPX4 at various time points after IRI. The results showed that administration of RR-11a abolished the increases of Acsl4 and Cox-2 expression and suppression of GPX4 at 1, 2, and 7 days after IRI (Fig. 7J, $\mathrm{K})$. Therefore, our data show that the inhibitor of legumain attenuates tubular injury and ferroptosis induced by IRI.

\section{Discussion}

Progress on developing therapeutic strategies that improve AKI outcomes remains slow, although a number of candidate molecular targets for effective therapies to prevent or ameliorate AKI have been identified in recent years. Our study showed that depletion of legumain, a conserved lysosomal endopeptidase, protects against the tubular injury in AKI caused by IRI or nephrotoxic folic acid. Moreover, we provide evidences that the beneficial effect of legumain deficiency correlates with suppression of tubular ferroptosis.

As a unique pattern of programmed cell death different from apoptosis, ferroptosis has been shown to be a promising therapeutic target for kidney diseases, especially those characterized by tubular death, including $\mathrm{AKI}^{19,20}$. Novel small molecules that specifically block ferroptosis, including ferrostatin-1 and liproxstatin-1, protect isolated renal tubules from erastin- and iron loading-induced cell death, and reduce kidney injury following acute oxalateinduced damage and in a model of severe kidney $\mathrm{IRI}^{21}$. 


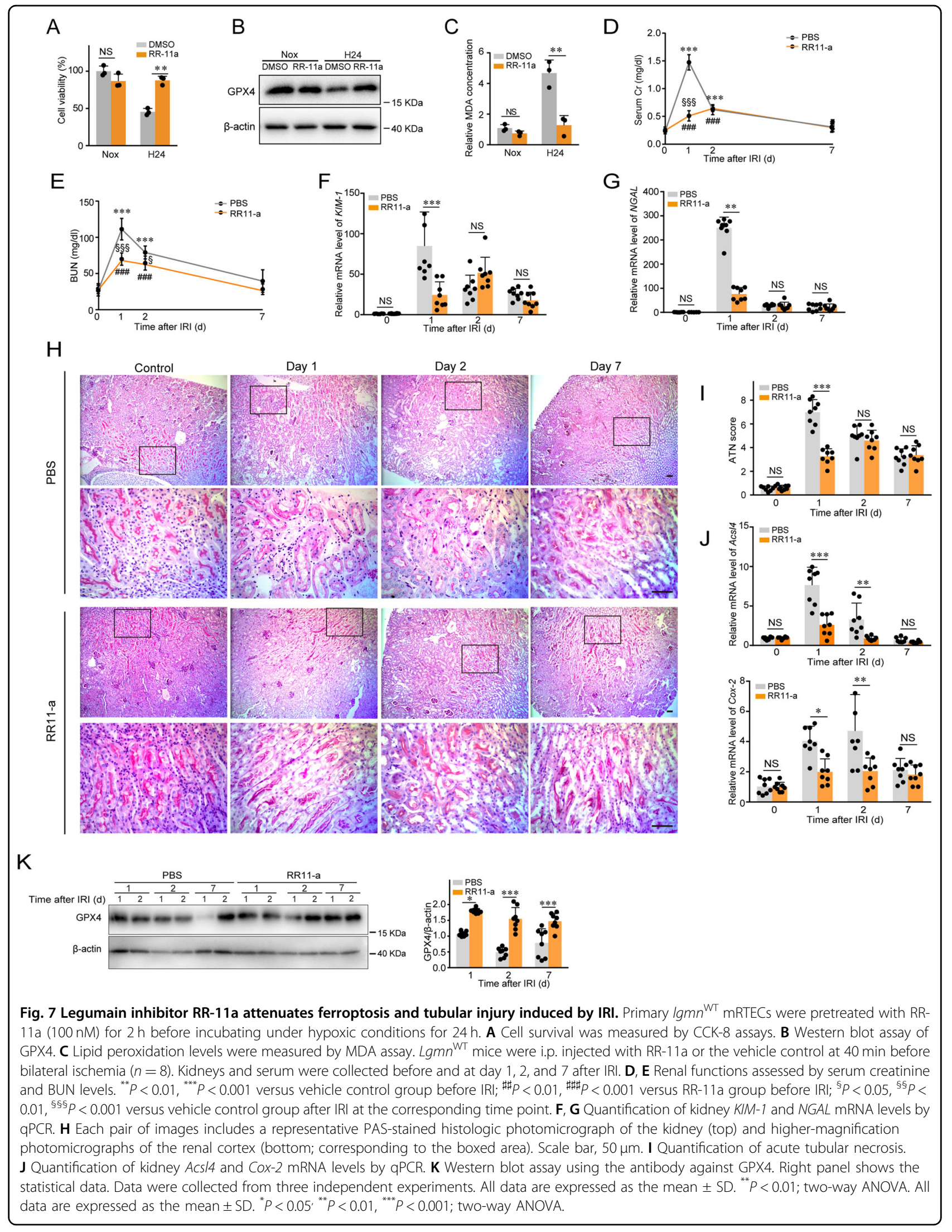


Compared with other forms of regulated cell death, ferroptosis results from inactivation of an essential metabolic process, leading to iron-catalyzed, lipid reactive oxygen species-mediated cellular collapse ${ }^{14}$. Cells undergoing ferroptosis are likely to release several immune modulators that are beneficial to trigger immune responses ${ }^{5,7}$. In this study, compared with wildtype control, IRI induced less infiltrated inflammatory cells and immunostimulatory signals in legumain knockout mice, which were accompanied by inhibition of ferroptosis. Thus, these data suggest that the renal beneficial effect of legumain knockout was not only due to the decrease of tubular cell loss, but also suppressed inflammation that amplifies injury responses and repair. It is noteworthy that studies have implicated distinct forms of regulated necrosis in AKI, indicating the necessity of combined therapies that block multiple pathways of regulated cell death simultaneously ${ }^{2,10}$.

Legumain is a member of the clan CD family $\mathrm{C} 13$ of cysteine proteases because of recognition of a conserved $\mathrm{His}^{148}$-Gly-spacer-Ala-Cys ${ }^{189}$ motif $^{22}$. It has been shown to be involved in various pathological situations including cancer $^{23}$, neurodegenerative disorders ${ }^{24}$, and kidney diseases ${ }^{18}$. The canonical translocation pathway of legumain is routed from the endoplasmic reticulum and Golgi to the endosome before it becomes activated in lysosomes ${ }^{17}$. However, legumain is also found at other "non-canonical" compartments outside of acidic vesicles, such as the cytoplasm, nucleus, and cell surface, implying diversity of its functions ${ }^{25}$. Loss of legumain induces proliferative abnormalities in the murine kidney due to accumulation of low molecular weight proteins in lysosomes, especially EGF receptor ${ }^{18}$. However, tubular cell- and macrophage-derived legumain has been reported to participate in remodeling of the extracellular matrix in the obstructive nephropathy model of unilateral ureteral obstruction ${ }^{26,27}$. In neurodegenerative disorders, the effects of legumain are mediated by cleavage of Tau and $\alpha$-synuclein in Alzheimer's and Parkinson's diseases ${ }^{24,28,29}$. Studies in tumor condition indicate that cytosolic legumain interacts with E3 ligase TRAF6 and activates lys63-linked ubiquitination. This ubiquitination results in ternary complex formation with heat shock protein 90 (HSP90), thereby increasing intracellular legumain stability and secretion ${ }^{30}$. The concentration of legumain in circulation correlates positively with the malignancy of breast cancer patients ${ }^{30}$. The exact roles of cytosolic legumain in distinct contexts are still unclear since the $\mathrm{pH}$ value of the cytosol is obviously unsuitable for the maturation of enzymatic legumain. In the present study, we found that cytosolic legumain interacted with the ferroptosis inhibitor GPX4, thereby promoting ferroptosis by increasing lysosomal autophagy of GPX4.

As an enzyme crucial to convert toxic lipid hydroperoxides into nontoxic lipid alcohols, GPX4 has been thought to be the main controller of ferroptosis ${ }^{12,31}$. Inactivation of GPX4 by direct inhibition with RSL3 or depletion of GPX4 results in overwhelming lipid peroxidation and cell death ${ }^{32,33}$. A recent study showed that chaperone-mediated autophagy is involved in the execution of ferroptosis, and HSP90 acts as a common regulatory mechanism shared by necroptosis and ferroptosis ${ }^{16}$. Here, our data indicated interactions of legumain, GPX4, and HSP90 in the cytoplasm. In addition, knockout of legumain impeded lysosomal transport and subsequent autophagy of GPX4 in erastin- and hypoxia-induced ferroptosis of tubular cells. In different AKI models, autophagy is rapidly induced in proximal tubular cells ${ }^{34}$. Moreover, it has been implicated in tubular atrophy observed in post-AKI tubules ${ }^{35,36}$. Pharmacological or genomic blockade of autophagy induction in AKI enhances kidney injury ${ }^{37-39}$. At present, the effect of autophagy on kidney injury during AKI is still controversial. It is possible that the timing of autophagy is important to determine its beneficial or deleterious effects. Further study is obviously needed to elucidate the role of this process of degradation and recycling of intracellular substances in tubular injury caused by AKI.

In conclusion, our findings reveal that legumain is involved in the pathogenesis of AKI by participating in chaperone-mediated autophagy of critical ferroptosisprotective factor GPX4, suggesting legumain a therapeutic target and early diagnostic marker for AKI.

\section{Materials and methods \\ Chemical reagents}

Erastin, ferrostatin-1 (Fer-1), and necrostatin-1 (Nec-1) were purchased from MedChem Express (Monmouth Junction, NJ). Chloroquine (CQ) and bafilomycin A1 (Baf A1) were purchased from Selleck Chemicals (Houston, TX). MG-132, deferoxamine (DFO), and folic acid were obtained from Sigma-Aldrich (St. Louis, MO). RR-11a was purchased from Wuxi Apptech (Beijing, China).

\section{Animal models}

The experimental animals were provided by Cyagen Biosciences, Inc. (Suzhou, China). The $\operatorname{lgm} n^{\mathrm{KO}}$ mice were generated using homologous recombination methods. The genetic background of embryonic stem cells and the Flp mice used in this experiment was C57BL/6. Offspring were genotyped by PCR according to the manufacturer's protocol (Cyagen). Littermates wild type mice were used as control mice $\left(\operatorname{lgmn} n^{\mathrm{WT}}\right)$. Experiments were performed in accordance with the Institutional Animal Use and Care Committee of Nankai University. Two AKI models were established as follows. Mice were randomly separated into experimental groups and control groups. (1) Bilateral IRI: mice (male, $8-10$ weeks old) on the $\operatorname{lgm} n^{\mathrm{KO}}$ background or littermate control mice were anesthetized by an 
intraperitoneal (i.p.) injection of chloral hydrate and placed on a warm pad to retain their body temperature. A bilateral flank incision was made, both sides of the renal vessels were occluded with clamps for $40 \mathrm{~min}$ followed by removing the clamps to induce blood reperfusion. The same procedure was performed in the control group without vessel clamping. (2) Nephrotoxic folic acidinduced AKI: mice (female, 12-14 weeks old) received a single i.p. injection of folic acid at $250 \mathrm{mg} / \mathrm{kg}$ in $0.3 \mathrm{~mol} / \mathrm{L}$ sodium bicarbonate or the vehicle. For therapeutic experiments, RR-11a was freshly dissolved in saline. Mice were administered an i.p. injection of $20 \mathrm{mg} / \mathrm{kg} R R-11 \mathrm{a}$ or the vehicle before ischemia. Mice were euthanized at the indicated times to collect serum samples and kidney tissues.

\section{Assessment of kidney functions and histology}

Concentrations of BUN and serum creatinine were measured using a urea and creatinine assay kit (Nanjing Jiancheng Bioengineering Institute, Nanjing, China). Periodic acid-Schiff (PAS) staining (Solarbio, Beijing, China) was performed according to the manufacturer's instructions. Immunohistochemistry and immunofluorescence staining were performed as described previously ${ }^{27}$. For immunohistochemistry, an antibody against CD3 (1:200, sc-20047; Santa Cruz Biotechnology, Inc., Dallas, TX) was used. Sections were counterstained with hematoxylin. Quantification was conducted in 15 randomly chosen fields $(\times 400)$, and the percentages of the positively stained areas were calculated. For immunofluorescence staining, a primary anti-F4/80 antibody (1:200, ab6640; Abcam, Inc., Cambridge, MA) was used. Nuclei were stained with DAPI and the specimens were mounted in fluorescence mounting medium (Dako, Carpinteria, CA). The specimens were analyzed by confocal microscopy (FV1000, Olympus Corp., Tokyo, Japan). The number of F4/80 macrophages was counted in 15 randomly chosen fields $(\times 400)$.

\section{Evaluation of renal damage}

PAS-stained kidney specimens were used to evaluate renal damage, tubular detachment, tubular dilation, protein cast, and the brush border damage were quantitated in 10 randomly selected fields (original magnification, $\times 200$ ). Percentage of areas per whole section are presented. Evidence of tubular detachment, proximal tubular dilation, and brush border damage were scored from zero to $10(0-1$, none; $1-2,<11 \%$; $2-4,11-25 \%$; $4-6,26-45 \%$; $6-8,46-75 \% ; 8-10,>75 \%)^{40}$. Results for each item were added to yield the ATN score.

\section{RNA extraction and quantitative real-time PCR}

Total RNA was extracted using TRIzol Reagent (Invitrogen, Carlsbad, CA), according to the manufacturer's instructions, and $2 \mu \mathrm{g}$ RNA was reverse transcribed with TransScript First-Strand cDNA synthesis SuperMix (TransGen Biotech, Beijing, China). Quantitative PCR was performed with TransStart Top Green qPCR SuperMix (TransGen Biotech) in a CFXTM Real-Time Thermal cycler (Bio-Rad, Hercules, CA). Primers are described in the supplemental material. Data were analyzed by the $2^{-\Delta \Delta \mathrm{Ct}}$ method, normalized to $\beta$-actin, and compared with controls.

\section{Mouse primary tubule isolation and cell culture}

Kidneys were collected immediately after the mice were euthanized, and cortexes were minced and digested for 1 hour using $1 \mathrm{mg} / \mathrm{ml}$ collagenase IV (Sigma-Aldrich). The tissue homogenate was filtered through $40-\mu \mathrm{m}$ and then $70-\mu \mathrm{m}$ strainers (BD Falcon, San Jose, CA) twice. Samples were centrifuged to obtain a pellet containing renal tubules. Tubules were cultured in DMEM/F12 medium containing $25 \mathrm{ng} / \mathrm{ml}$ mouse epithelial growth factor (Sino Biological, Beijing, China). Human proximal tubular cell line HK-2 was purchased from the ATCC (Manassas, VA) and cultured in DMEM/F12 medium containing $10 \% \mathrm{FBS}$ and $1 \%$ penicillin and streptomycin (Gibco, Gaithersburg, MD). Cells were incubated in a hypoxic $\left(0.001 \% \mathrm{O}_{2}\right)$ chamber for 6,12 , and 24 hours to mimic ischemic conditions in vivo.

\section{Western blotting}

Kidney tissues and cell pellets were homogenized in $1 \times$ radioimmunoprecipitation assay buffer containing protease inhibitor cocktails, and the insoluble residue was removed by centrifugation for 10 minutes at $12,000 \times g$. The protein concentration was determined using the Bradford assay (Thermo Fisher Scientific, Waltham, MA). Equal amounts of proteins were separated by $10-15 \%$ SDS-PAGE. Samples were transferred to polyvinylidene difluoride membranes (EMD Millipore, Billerica, MA), blocked with $5 \%$ dry skim milk, and incubated with primary antibodies including anti-legumain (1:1000; AF2058; R\&D Systems, Minneapolis, MN), anti-legumain (1:5000, ab183028), anti-GPX4 (1:2000, ab125066), anti-IL-33 (1:1000, alx-804-840-C100, Enzo Life Sciences, Plymouth Meeting, PA), TNF- $\alpha$ (1:1000, sc-12744, Santa Cruz Biotechnology, Inc.), anti-CCL-2 (1:1000, sc-52701, Santa Cruz Biotechnology, Inc.), anti-phospho-MLKL (1:1000; ab196436; Abcam, Inc.), mouse anti- $\beta$-actin (1:5000, sc-47778, Santa Cruz Biotechnology, Inc.), rabbit anti- $\beta$-actin (1:1000, sc-1616-R), anti-LAMP1 (1:1000; sc17768; Santa Cruz Biotechnology, Inc.), anti-HSP90 (1:1000; WL01763; Wanleibio, Shenyang, China), antiHSC70 (1:1000, 10654-1-AP, ProteinTech Group, Chicago, IL, USA), anti-lamp-2a (1:1000, 66301-1-Ig, ProteinTech Group) or anti-caspase 3 (1:1000; $9662 \mathrm{~s}$; Cell Signaling Technology, Beverly, MA) at $4{ }^{\circ} \mathrm{C}$ overnight. 
After washing with TBS/Tween, the membranes were incubated with a horseradish peroxidase-conjugated secondary antibody (Santa Cruz Biotechnology, Inc.). Blots were developed with an ECL kit (EMD Millipore).

\section{Detection of intracellular ROS}

Two kinds of fluorescent probes were used to detect intracellular ROS in kidney tissues and cells, namely 5(and-6)-carboxy-2', $7^{\prime}$-dichlorodihydrofluorescein diacetate (carboxy- $\mathrm{H}_{2}$ DCFDA; Sigma-Aldrich), and dihydroethidium (DHE; Sigma-Aldrich). For DHE staining, cryosections were incubated with $10 \mu \mathrm{M}$ DHE at $37^{\circ} \mathrm{C}$ for $30 \mathrm{~min}$ and then observed by confocal microscopy to determine the percentage of the DHE-stained area. For carboxy-H2DCFDA staining, kidney tissues were homogenized with PBS and centrifuged. Then, the supernatant was collected and incubated with $10 \mu \mathrm{M} \mathrm{H}_{2}$ DCFDA at $37^{\circ} \mathrm{C}$ for $30 \mathrm{~min}$. For ROS detection in cultured cells, the cells were seeded in a 96-well plate and incubated with $\mathrm{H}_{2}$ DCFDA. Fluorescence was detected by a SpectraMax Microplate Reader (Molecular Devices, San Jose, CA) at $488 \mathrm{~nm}$ excitation and $525 \mathrm{~nm}$ emission.

\section{MDA assay}

The relative MDA concentration in kidney tissues and cells was assessed with a lipid peroxidation MDA assay kit (Beyotime, Shanghai, China), according to the manufacturer's instructions. Briefly, kidney tissues or cells were homogenized in lysis buffer, incubated with thiobarbituric acid at $100^{\circ} \mathrm{C}$ for $15 \mathrm{~min}$, centrifuged, and then absorbance was determined at $532 \mathrm{~nm}$.

\section{Cell survival and LDH release assays}

Cell survival was determined using a cell counting kit-8 (CCK-8) assay (Dojindo Laboratories, Kumamoto, Japan). mRTECs and HK-2 cells were seeded in 96-well plates at 3000 cells per well and treated with erastin or incubated under hypoxic conditions. Subsequently, $110 \mu \mathrm{l}$ of fresh medium containing $10 \mu \mathrm{l} \mathrm{CCK-8} \mathrm{solution} \mathrm{was} \mathrm{added} \mathrm{to}$ the cells, followed by incubation for $2 \mathrm{~h}\left(37^{\circ} \mathrm{C}, 5 \% \mathrm{CO}_{2}\right)$. Absorbance at $450 \mathrm{~nm}$ was then measured. $\mathrm{LDH}$ release was detected using a lactate dehydrogenase (LDH) kit (Nanjing Jiancheng Bioengineering Institute). mRTECs were seeded in 6-well plates at $1 \times 10^{5}$ cells per well and treated with erastin or incubated under hypoxic conditions. The culture supernatant was collected and the cells were treated with $1.5 \%$ Triton X-100, and then the cells and supernatant were incubated with coenzyme I and 2,4dinitrophenylhydrazine for $15 \mathrm{~min}$ at $37^{\circ} \mathrm{C}$. Absorbance was then determined at $490 \mathrm{~nm}$.

\section{Lysosome isolation}

Lysosomes were harvested by homogenization and sequential centrifugation with a lysosome isolation kit
(BestBio, Shanghai, China), according to the manufacturer's protocol.

\section{Plasmid construction and transfection}

Mouse legumain cDNA was amplified by reverse transcription-PCR and then ligated into pLV-EF1 $\alpha$-MCSIRES-Bsd (Biosettia, San Diego, CA). mRTECs were transfected with the pLV-EF1 $\alpha$-legumain-IRES-Bsd plasmid. Cells were seeded in a 6-well plate containing medium without penicillin-streptomycin and then transfected using Lipofectamine ${ }^{\mathrm{TM}} 2000$ transfection reagent (Invitrogen).

\section{Immunoprecipitation}

Cell pellets were lysed in lysis buffer and incubated with an antibody against legumain (312114; Invitrogen) at $4^{\circ} \mathrm{C}$ overnight. Immunocomplexes were captured using protein G-sepharose (GE Healthcare, Buckinghamshire, UK) for $3-5 \mathrm{~h}$ at $4{ }^{\circ} \mathrm{C}$. The beads were washed with PBS five times, and then a loading buffer was used to elute the immunocomplexes.

\section{Statistical analyses}

Data were analyzed by GraphPad Prism, ver.7.0 (GraphPad Software Inc. La Jolla, CA) and shown as the mean \pm SD. The unpaired Student's t-test was used to compare two groups. ANOVA was used to compare more than two groups.

\section{Acknowledgements}

The National Natural Science Foundation of China is gratefully acknowledged.

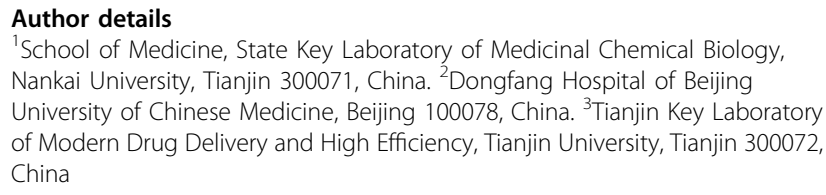

\section{Author contributions}

X.T. designed the study; C.C., Y.Y., D.W., T.Z., N.M., Y.W., L.K., Y.Z., and L.D. carried out experiments; X.T., C.C., and D.W. analyzed the data; X.T., C.C., M.Z., J.G., Z.Z., Y.Z., and X.M. made the figures; X.T., C.C., and S.Y. drafted and revised the manuscript; all authors approved the final version of the manuscript.

\section{Funding statement}

This work was supported by the National Natural Science Foundation of China grant 81872254 (X.T.).

\section{Ethics statement}

Animal researches were executed in compliance with the Institutional Animal Use and Care Committee of Nankai University.

Conflict of interest

The authors declare that they have no conflict of interest.

\section{Publisher's note}

Springer Nature remains neutral with regard to jurisdictional claims in published maps and institutional affiliations. 
Supplementary Information accompanies this paper at (https://doi.org/ 10.1038/s41419-020-03362-4).

Received: 13 July 2020 Accepted: 1 December 2020

Published online: 11 January 2021

\section{References}

1. Bellomo, R., Kellum, J. A. \& Ronco, C. Acute kidney injury. Lancet 380, 756-766 (2012).

2. Linkermann, A. et al. Regulated cell death in AKI. J. Am. Soc. Nephrol. 25 2689-2701 (2014)

3. Siew, E. D. et al. Predictors of Recurrent AKI. J. Am. Soc. Nephrol. 27, 1190-1200 (2016).

4. Rewa, O. \& Bagshaw, S. M. Acute kidney injury-epidemiology, outcomes and economics. Nat. Rev. Nephrol. 10, 193-207 (2014).

5. Linkermann, A., Stockwell, B. R., Krautwald, S. \& Anders, H. J. Regulated cell death and inflammation: an auto-amplification loop causes organ failure. Nat. Rev. Immunol. 14, 759-767 (2014).

6. Pefanis, A., lerino, F. L., Murphy, J. M. \& Cowan, P. J. Regulated necrosis in kidney ischemia-reperfusion injury. Kidney Int. 96, 291-301 (2019).

7. Xie, Y. et al. Ferroptosis: process and function. Cell Death Differ. 23, 369-379 (2016).

8. Yang, W. S. \& Stockwell, B. R. Ferroptosis: death by lipid peroxidation. Trends Cell Biol. 26, 165-176 (2016).

9. Dixon, S. J. et al. Ferroptosis: an iron-dependent form of nonapoptotic cell death. Cell 149, 1060-1072 (2012).

10. Linkermann, A. et al. Synchronized renal tubular cell death involves ferroptosis. Proc. Natl Acad. Sci. USA 111, 16836-16841 (2014).

11. Martin-Sanchez, D. et al. Ferroptosis, but not necroptosis, is important in nephrotoxic folic acid-induced AKI. J. Am. Soc. Nephrol. 28, 218-229 (2017).

12. Yang, W. S. et al. Regulation of ferroptotic cancer cell death by GPX4. Cell 156, 317-331 (2014).

13. Friedmann Angeli, J. P. et al. Inactivation of the ferroptosis regulator Gpx4 triggers acute renal failure in mice. Nat. Cell Biol. 16, 1180-1191 (2014).

14. Stockwell, B. R. et al. Ferroptosis: a regulated cell death nexus linking metabolism, redox biology, and disease. Cell 171, 273-285 (2017).

15. Zou, Y. et al. A GPX4-dependent cancer cell state underlies the clear-cell morphology and confers sensitivity to ferroptosis. Nat. Commun. 10, https:// doi.org/10.1038/s41467-019-09277-9 (2019).

16. $\mathrm{Wu}$, Z. et al. Chaperone-mediated autophagy is involved in the execution of ferroptosis. Proc. Natl Acad. Sci. USA 116, 2996-3005 (2019).

17. Dall, E. \& Brandstetter, H. Structure and function of legumain in health and disease. Biochimie 122, 126-150 (2016).

18. Miller, G., Matthews, S. P., Reinheckel, T., Fleming, S. \& Watts, C. Asparagine endopeptidase is required for normal kidney physiology and homeostasis. FASEB J. 25, 1606-1617 (2011).

19. Doll, S. et al. FSP1 is a glutathione-independent ferroptosis suppressor. Nature 575, 693-698 (2019).

20. Skouta, R. et al. Ferrostatins inhibit oxidative lipid damage and cell death in diverse disease models. J. Am. Chem. Soc. 136, 4551-4556 (2014).

21. Cao, J. Y. \& Dixon, S. J. Mechanisms of ferroptosis. Cell. Mol. Life Sci. 73 , 2195-2209 (2016).
22. Chen, J. M., Rawlings, N. D., Stevens, R. A. \& Barrett, A. J. Identification of the active site of legumain links it to caspases, clostripain and gingipains in a new clan of cysteine endopeptidases. FEBS Lett. 441, 361-365 (1998).

23. Liu, C., Sun, C. Z., Huang, H. N., Janda, K. \& Edgington, T. Overexpression of legumain in tumors is significant for invasion/metastasis and a candidate enzymatic target for prodrug therapy. Cancer Res. 63, 2957-2964 (2003).

24. Zhang, Z. et al. Cleavage of tau by asparagine endopeptidase mediates the neurofibrillary pathology in Alzheimer's disease. Nat. Med. 20, 1254-1262 (2014).

25. Dall, E. \& Brandstetter, H. Mechanistic and structural studies on legumain explain its zymogenicity, distinct activation pathways, and regulation. Proc Natl Acad. Sci. USA 110, 10940-10945 (2013).

26. Morita, Y. et al. Legumain/asparaginyl endopeptidase controls extracellular matrix remodeling through the degradation of fibronectin in mouse renal proximal tubular cells. FEBS Lett. 581, 1417-1424 (2007).

27. Wang, D. et al. Legumain, an asparaginyl endopeptidase, mediates the effect of M2 macrophages on attenuating renal interstitial fibrosis in obstructive nephropathy. Kidney Int. 94, 91-101 (2018).

28. Kang, S. S. et al. alpha-Synuclein stimulation of monoamine oxidase-B and legumain protease mediates the pathology of Parkinson's disease. EMBO J. 37, https://doi.org/10.15252/embj.201798878 (2018).

29. Zhang, Z. et al. Asparagine endopeptidase cleaves alpha-synuclein and mediates pathologic activities in Parkinson's disease. Nat. Struct. Mol. Biol. 24, 632-642 (2017)

30. Lin, Y. et al. Functional role of asparaginyl endopeptidase ubiquitination by TRAF6 in tumor invasion and metastasis. J. Natl Cancer Inst. 106, dju012 (2014).

31. Imai, H., Matsuoka, M., Kumagai, T., Sakamoto, T. \& Koumura, T. Lipid peroxidation-dependent cell death regulated by GPX4 and ferroptosis. Curr. Top. Microbiol. Immunol. 403, 143-170 (2017).

32. Bruni, A. et al. Ferroptosis-inducing agents compromise in vitro human islet viability and function. Cell Death Dis. 9, https://doi.org/10.1038/s41419-0180506-0 (2018)

33. Doll, S. et al. ACSL4 dictates ferroptosis sensitivity by shaping cellular lipid composition. Nat. Chem. Biol. 13, 91-98 (2017).

34. Kaushal, G. P. \& Shah, S. V. Autophagy in acute kidney injury. Kidney Int. $\mathbf{8 9}$ 779-791 (2016)

35. Li, L., Zepeda-Orozco, D., Black, R. \& Lin, F. Autophagy is a component of epithelial cell fate in obstructive uropathy. Am. J. Pathol. 176, 1767-1778 (2010).

36. Koesters, R. et al. Tubular overexpression of transforming growth factor-beta1 induces autophagy and fibrosis but not mesenchymal transition of renal epithelial cells. Am. J. Pathol. 177, 632-643 (2010).

37. Jiang, M., Liu, K., Luo, J. \& Dong, Z. Autophagy is a renoprotective mechanism during in vitro hypoxia and in vivo ischemia-reperfusion injury. Am. J. Pathol. 176, 1181-1192 (2010).

38. Periyasamy-Thandavan, S. et al. Autophagy is cytoprotective during cisplatin injury of renal proximal tubular cells. Kidney Int. 74, 631-640 (2008).

39. Jiang, $\mathbf{M}$. et al. Autophagy in proximal tubules protects against acute kidney injury. Kidney Int. 82, 1271-1283 (2012).

40. Arai, S. et al. Apoptosis inhibitor of macrophage protein enhances intraluminal debris clearance and ameliorates acute kidney injury in mice. Nat. Med. $\mathbf{2 2}$ 183-193 (2016). 